\title{
Beam Deflection for Temporal Encoding in Time-of-Flight Mass Spectrometry
}

\author{
G. E. Yefchak, G. A. Schultz, J. Allison, and C. G. Enke \\ Department of Chemistry, Michigan State University, East Lansing, Michigan, USA \\ J. F. Holland \\ Department of Biochemistry, Michigan State University, East Lansing, Michigan, USA
}

\begin{abstract}
The pulsed ion sources used in conventional time-of-flight mass spectrometry (TOFMS) generally do not provide adequate resolving power across the mass range required for applications such as gas chromatography combined with mass spectrometry (GC/MS). Theoretical and experimental aspects of beam deflection techniques, which provide time encoding for TOFMS with continuous ions sources, are explored here. In this approach, ion source conditions do not affect resolving power, allowing for a greater variety of ionization modes to be used. Theoretical predictions for the resolving power attainable with beam deflection, which are satisfactory for GC/MS applications, agree well with experimentally determined values. The combination of GC-beam deflection-TOFMS with time-array detection is evaluated, and the capabilities of this system are compared to those of scanning mass spectrometers. (f Am Soc Mass Spectrom 1990, 1, 440-447)
\end{abstract}

$\mathrm{A}$ Il varieties of time-of-flight (TOF) mass spectrometers share a need for synchronized ion packet production. The departure of ion packets from the ion source serves to indicate the starting times for ion velocity measurements, and the dimension of the ion packet determines mass resolving power. Although most TOF mass spectrometers make use of so-called pulsed ion sources (i.e., ion sources that generate ion packets directly), there are a number of circumstances in which it is desirable to use sources that produce ions continuously. Beams produced by these "continuous ion sources," which are similar to sources used in magnetic sector and quadrupole mass spectrometers, are spatially modulated as described below to create the desired ion packets for TOF mass analysis.

A theoretical treatment of beam modulation techniques in nuclear physics appeared in 1960 [1], and in 1973 many theoretical considerations for beam modulation in TOF mass spectrometry (TOFMS) were elegantly described by Bakker [2]. Shortly thereafter, Bakker reported the construction of a beam modulation TOF mass spectrometer and an experimental confirmation of the theory [3]. Various other TOF instruments incorporating beam modulation have since been reported, including those of Pinkston et al. [4], Glish and Goeringer [5], and Eckenrode et al. [6]. A beam modulation ion source was also developed for the early Bendix TOF spectrometers by Futrell and coworkers $[7,8]$. In addition, a similar technique has

Address reprint requests to C. G. Enke, Department of Chemistry, Michigan State University, East Lansing, MI 48824. been used in a tandem TOF mass spectrometer to select parent ions prior to a second stage of mass analysis [9]. The "orthogonal-acceleration" TOFMS instrument recently described by Dawson and Guilhaus [10] incorporates beam-deflection in the broadest sense, but the operating principle of orthogonal acceleration differs sufficiently from conventional beam-modulation techniques to exclude its discussion in this work.

Ion packet production by modulation of a continuous beam eliminates some of the difficulties encountered with more conventional pulsed-source techniques, particularly when gaseous samples such as those encountered in gas chromatography combined with mass spectrometry (GC/MS) are ionized. With pulsed ion sources, three patterns of ion behavior can lead to decreased mass resolving power $[11,12]$. The distribution of initial positions of ions within the source, especially important for gaseous samples, and the distribution of initial ion velocities both lead to a spread in final kinetic energy. This spread in kinetic energy leads to a distribution of arrival times for isomass ions and hence to the loss of resolving power. Furthermore, the thermal motion of ions moving with equal speeds toward and away from the source exit prior to the application of the extraction field results in the "turn-around" separation between isomass ions ultimately having identical on-axis energies. The deterioration of resolving power due to this turn-around problem has normally been most severe, necessitating the use of high extraction field gradients that minimize the turn-around time in pulsed ion sources. Unfortunately, the use of large field gradients exacerbates the spatial problem; this incompatibility between space 
focusing and energy focusing with gaseous samples has been described in the literature $[11,13]$. Production of ion packets by modulation of continuous beams eliminates the turn-around problem because all ions in the accelerated beam are moving in the same direction. Furthermore, this elimination of the turn-around effect permits low extraction gradients to be used, which significantly decrease the energy spread caused by the initial spatial distribution. For these reasons, studies of beam modulation have been undertaken in an effort to achieve adequate mass resolving power in GC/MS with TOF instrumentation. Both the theoretical and experimental results reported here demonstrate that beam deflection TOFMS is a viable technique for GC/MS when used in combination with time-array detection.

\section{Methodology}

The beam deflection TOFMS used for this study was constructed by adapting the electron impact ion source from a Dupont 21-491B double-focusing mass spectrometer to a Bendix 12-101 TOFMS flight tube having a $2.1 \mathrm{~m}$ length. The ion source was operated at an accerating potential of $3 \mathrm{kV}$ in a continuous ion extraction mode. The ion beam enters the flight tube region where beam modulation takes place. The beam deflection assembly was constructed from copper-plated circuit boards and consists of two plates, $1.4 \mathrm{~cm}$ in length $\times 2.0 \mathrm{~cm}$ in height, separated by a distance of $1.3 \mathrm{~cm}$. This assembly was placed about $2 \mathrm{~cm}$ past the source exit slit. A Galileo CEMA detector was used to collect the ion current. The overall geometry of the instrument is shown in Figure 1.

Two dynamic voltages are used in tandem to modulate the continuous ion beam. Initially, the voltage applied to one of the plates is held at $+63 \mathrm{~V}$, while the voltage applied to the other plate is $0 \mathrm{~V}$. When beam modulation is desired, these voltage levels are switched, in approximately $10 \mathrm{~ns}$, to $0 \mathrm{~V}$ and $+63 \mathrm{~V}$, respectively. This is equivalent to the case in which one plate is held at ground potential throughout the experiment while the other plate is swept from -63

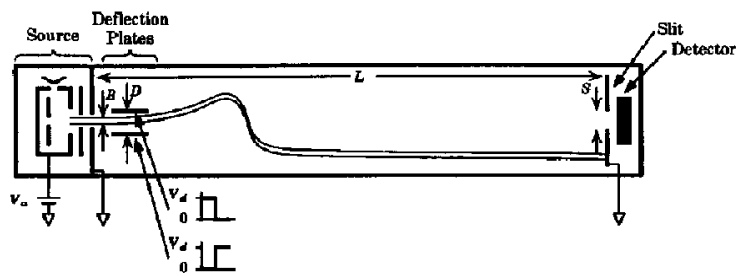

Figure 1. Schematic dlagram of the beam-deflection TOFMS instrument. The parameters of interest include the beam width, $B$, the deflection plate separation, $D$, the flight-path length, $L$, and the detector aperture width, $S$. Ions are accelerated by the voltage $V_{a}$ applied between the source block and the source exit slit. Beam deflection is accomplished by the voltage $V_{d}$ applied to the upper and lower deflection plates as shown (see text).
$\mathrm{V}$ to $+63 \mathrm{~V}$ (i.e., $V_{d}=63 \mathrm{~V}$ for the equations given below). The dual-voltage approach is used so that the magnitude of the field reversal can be twice as great, for the same rise time, as would be possible with only one pulsed voltage. The new voltage applied to each plate is maintained for $100 \mu \mathrm{s}$, which allows all of the ion packets to strike the detector surface before the next ion modulation occurs. This modulation generates a new set of ion packets, but the signal produced by these ions is ignored by the detection equipment so that only transients generated by every other modulation are collected. This process, which allows a 5$\mathrm{kHz}$ sampling rate, is used in order to avoid difficulties caused by asymmetry between the rising and falling edges of the deflection pulses. The beam modulation circuitry was designed in-house; schematic diagrams for the circuits may be obtained from the authors.

Computer programs used for the simulations were written in VAX-11 C and run on a Digital Equipment Corporation VAXstation 3200 computer (Maynard, MA). The animation program was written in Microsoft QuickC (MicroSoft, Redmond, WA) and run on an IBM-AT compatible computer.

\section{Theory}

\section{Models for Beam Modulation Techniques}

The ideal desired behavior of all beam modulation techniques is illustrated in Figure 2; a packet having an arbitrarily short temporal width is "chopped" out of a continuous beam. Note that the packet retains the kinetic energy distribution present in the original beam and is finite in spatial and temporal width; these are the only nonidealities if the fringing fields near the ends of the deflection plates are ignored. All ions depicted in Figure 2 and throughout this work have the same mass-to-charge ratio. In actual operation, of course, an instrument would produce ions of various masses. Ion trajectories in a beam deflection instrument are independent of mass, though the velocities of the different isomass packets are mass-dependent.

Modulation techriques have so far all relied upon deflection of the beam by an electric field, generated by some voltage function $V(t)$, applied across a pair of deflection plates between which the ions pass. Some portion of the beam is deflected across an aperture, causing an ion packet to emerge from the opposite side. Various $V(t)$ functions may be used to generate ion packets via at least three modes of ion behavior. The ion behavior patterns corresponding to these modes

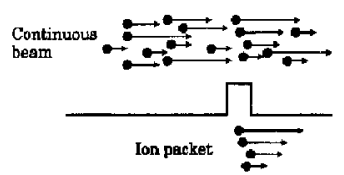

Figure 2. Ideal ion behavior in beam modulation; the process essentially yields a slice from the continuous beam. Arrow lengths indicate relative ion velocities; only isomass ions are considered. 

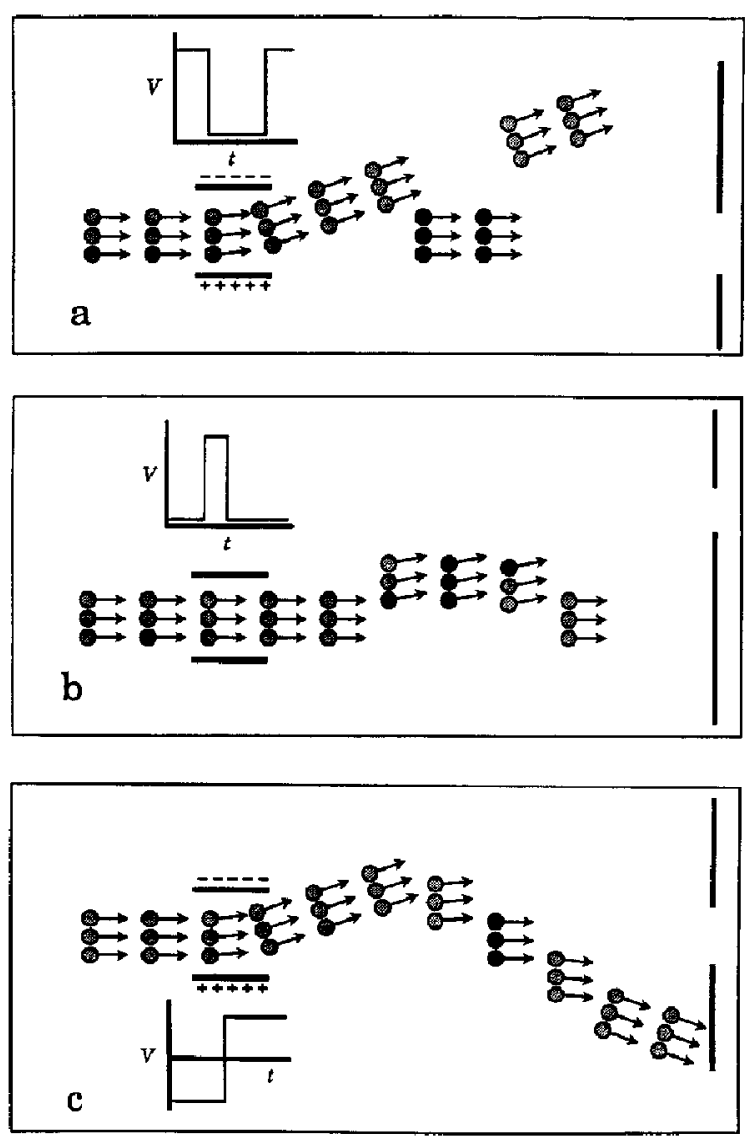

Figure 3. Three models for beam modulation techniques. Isomass ions are shown to pass through a pair of deflection plates and move toward an aperture. Relative duration and polarity of the deflection voltage pulse is indicated for each case. The darker shading indicates ions that will pass through the aperture and form the ion packet. (a) Gate model; (b) Impulse Sweep model; (c) Differential Impulse Sweep model.

are illustrated in Figure 3 . These include a pattern referred to here as the Gate mode, as well as the Impulse Sweeping and Differential Impulse Sweeping modes that were identified by Fowler and Good [1]. In each of these modes, the "default" condition, which exists before and after application of the packet-forming voltage pulse, is the deflection of the ion beam away from the aperture. In the Gate mode (Figure 3a) the field changes to allow the beam to pass directly through the aperture for some period of time; at the end of this time the field returns to its original state. In this mode $V(t)$ serves most closely as a "gate," either preventing or allowing passage of ions through the aperture. The Impulse Sweep mode (Figure $3 b$ ) is sirnilar to the Gate mode in that the field is pulsed "on" for a period of time and then returned to its original value. In Impulse Swceping, however, the deflection voltage pulse is short in duration, compared to the flight time through the plates. The pulse serves to add a velocity component perpendicular to the direction of ion motion; the resulting angular trajectories cause part of the beam to be swept into the aperture. Here the width of the emerging ion packet is related, but not equal, to the duration of the deflection pulse. The width and ion trajectories are also mass-dependent in this mode. Impulse Sweeping has been difficult to accomplish because it requires precise geometrical designs as well as a voltage impulse of precisely defined amplitude. Furthermore, it does not appear to have any advantage over the other two modes, so it will not be discussed further here. In the Differential Impulse Sweep mode (Figure 3c), the deflection voltage switches polarity, causing a "kink" to form in the beam. The portion of this kink that continues to move parallel to the original beam passes through the aperture to form the packet. A referee has noted that a type of Differential Impulse Sweeping could also be accomplished with unequal switching voltages.

The Gate mode is the simplest case. Here the width of the ion packet is essentially equal to the duration of the deflection pulse (neglecting the intermediate ion behavior that occurs when the voltage change takes place). Ions that pass through the plates while the deflection field is off continue onward and pass through the aperture; those that pass through before or after this pulse do not. In the description of this mode, the transient ion behavior caused by the voltage change itself is ignored; only the steady-state trajectories either through or away from the aperture are considered. Ion packets produced in the Gate mode are therefore always longer in duration than the flight time of the ions through the deflection plate region. This disadvantage may perhaps be offset by the fact that the Gate mode yields ion packets for which the temporal width is independent of mass.

The transient ion behavior that was ignored in the Gate mode is in fact responsible for packet formation in the Differential Impulse Sweep mode. This mode is therefore capable of producing much shorter ion packets. Consider ions that enter the deflection plate region just before the switch in the field direction. (The time under consideration here is somewhat earlier than that of the "snap shot" shown in Figure 3c.) These ions are deflected downward as they travel through the region. When the field changes polarity, however, they begin to experience an upward force. Those ions that have traveled half-way through the plate region when the pulse occurs will experience equal durations of downward and upward forces and thus exit the region moving parallel to their original trajectory. The aperture is assumed here to be located in line with the initial beam axis and the deflection plates. Some ions very near to the exact half-way point of the deflcction plate region at the time of the field reversal will also obtain final trajectories that allow for their passage through the aperture. The extent to which this occurs contributes to the temporal width of the packet and will be discussed in the following section. 


\section{Ion Packet Quality}

As derived by Bakker [2], the temporal base width of an ion packet resulting from the Differential Impulse Sweep process is given by the expression

$$
w_{I}=\frac{(B+S) D}{L V_{d}} \sqrt{\frac{V_{a} m}{2 e}}
$$

where $B$ is the beam width, $S$ is the aperture width, $D$ is the deflection plate separation, $L$ is the distance between the plates and the slit, and $V_{a}$ and $V_{d}$ are the acceleration and deflection voltages, respectively. (That is, the deflection voltage switches from $-V_{d}$ to $+V_{d}$.) The subscript in $w_{I}$ is meant to represent "ideal" because this is the width that would be obtained if the ion beam were ideal in terms of kinetic energy and angular dispersion. The principal assumptions for eq 1 are that the rise time for the voltage change is negligible and, more importantly, that the distance from the plates to the aperture, $L$, is much greater than the length of the plates. (Note, however, that the actual plate lengths do not appear in the expression.) This latter assumption implies that the aperture should not be placed near the deflection plates but rather at the detector. In fact, it is possible for the detector surface itself to serve as the aperture.

In addition to the ideal width $w_{1}$ derived by Bakker, the packet is broadened by the kinetic energy distribution of ions within the packet. Two ions leaving an initial position $x=0$ simultaneously, but with differing kinetic energies $U$ and $(1+\beta) U$, will arrive at a location $x=L$ separated in time by $\Delta t \approx \beta L(2 m / L)^{1 / 2} / 4$. Thus, an ion packet having negligible initial width but with a normal kinetic energy distribution having standard deviation $\sigma_{U}$ would be broadened, after traveling distance $L$, to have a half-width given approximately by

$$
w_{U}=\sqrt{\frac{2 m}{V_{a} e}} \frac{\beta L}{4}
$$

where $\beta=2.35 \sigma_{U} / U_{0}$.

A third cause for packet broadening was identified in this study for ion beams having nonzero angular divergence. Consider the two ions shown in Figure 4. The lower ion enters with no off-axis energy and is initially accelerated downward by the deflection field. As it reaches point $a$, half-way through the plate region, the field reverses and accelerates the ion in the upward dircetion. Since downward and upward force are experienced for equal periods of time, the ion leaves the region with no off-axis energy and thus is included in the final packet. The upper ion enters the region $\Delta t$ seconds prior to the lower ion and at an angle $\theta$ from the beam axis. Due to its off-axis energy, this ion continues to move upward for a period of time but is eventually deflected downward by the electric field. This ion experiences the downward force longer than

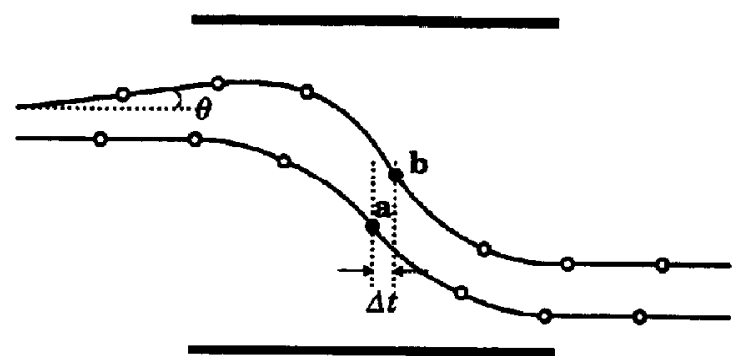

Figure 4. Two ions entering the deflection plate region with different trajectory angles. Field reversal occurs when the lower ion is at point and the upper ion is at point $b$.

the lower ion does because the upper ion travels to point $\mathbf{b}$ before the field reverses. This longer duration of the applied downward force, followed by a shorter duration of upward force (experienced from point b to the end of the deflection region), compensates for the initial off-axis energy. The ion therefore leaves the region parallel to the beam axis and is included in the final packet, despite being offset from the ideal ion by a time $\Delta t$.

The value of $\Delta t$ that satisfies these conditions may be related to off-axis angle, $\theta$, as follows. Consider an "ideal" (i.e., initially on-axis) ion traveling with the most-probable velocity $v_{0}=\left(2 V_{a} e / m\right)^{1 / 2}$. An ion entering the deflection plate region with an off-axis angle $\theta$ will have a velocity vector perpendicular to the beam axis given by $v_{y}=v_{0} \cos \theta$. When leaving the region, however, the perpendicular velocity $v_{y}^{\prime}$ is zero. If the ideal ion passes half-way through the plate region in time $t_{1 / 2}$, then the off-axis ion experiences downward and upward forces for times $t_{1 / 2}+\Delta t$ and $t_{1 / 2}-\Delta t$, respectively. The final perpendicular velocity of the offaxis ion is related to the initial value by the expression

$$
v_{f}^{\prime}=0=v_{0} \sin \theta-\frac{V_{d} e}{m D}\left(t_{1 / 2}+\Delta t\right)+\frac{V_{d} e}{m D}\left(t_{1 / 2}-\Delta t\right)
$$

which rearranges to $\Delta t=\frac{m D}{2 V_{d} e} \nu_{0} \sin \theta$. This represents the broadening of the peak to lower flight times due to ions with trajectories that diverge in the upward direction. (Only two-dimensional motion in the plane of Figure 4 is considered here.) For a true beam having a divergence half-angle of $\theta$, those ions having trajectory angles in the downward direction will cause similar broadening to higher flight times as well. Thus the quantity $2 \Delta t$ could be used to approximate the base width of a packet broadened only by this angular effect, yielding

$$
w_{\theta}=\frac{D}{V_{d}} \sqrt{\frac{2 V_{a} m}{e}} \sin \theta
$$

Equation 3 yields an underestimate of peak broadening due to the angular effect, however, because it is 
not necessary for ions to be moving exactly parallel to the beam axis as they leave the deflection region in order to pass through the aperture. Consider, for example, an ion that enters the deflection region with the same trajectory as the upper ion discussed above but slightly ahead of it in time. This ion would experience even more net downward force than the upper ion pictured in Figure 4, and would thus leave the region with a small downward velocity component. This final trajectory could, however, still carry the ion through the aperture (at a position somewhat below the point at which the pictured ion passes through). Ions acting in this way, as well as the analogous ions having initial downward motion, serve to further increase the final packet width. Though eq 3 cannot be used to obtain the complete magnitude of the peak broadening due to angular divergence, the digital simulation described below can be used to measure this effect.

The broadening caused by ions with positive and negative initial trajectory angles need not be symmetric because in both cases the forward velocity (in the direction of the beam axis) of these off-axis ions is reduced from the ideal velocity by a factor of $\cos \theta$ for ions having the same kinetic energy. The flight time for ions having an initial downward velocity component is increased over that of the ideal ion both by their lower velocity in the beam axis as well as the angular effect described above. For the inns having some initial upward motion, however, flight times tend to be decreased by the angular effect but increased by their reduced velocity. The net result of this is that the peak is skewed toward higher flight times.

It is difficult to combine the expressions for $w_{l}, w_{U}$, and $w_{\theta}$ directly to obtain an expression for true peak width, but any of the expressions can be used alone to approximate peak widths for cases in which one effect (geometry, energy distribution, or angular divergence) predominates. The combination of the three effects was explored by means of digital simulations as described below; the individual expressions were useful for checking the validity of these simulations as well.

\section{Simulations}

In order to explore the combination of the energy spread and angular divergence effects with the ideal packet width given by eq 1 , computer programs were developed to model ion packet behavior. First, an animation program was developed that illustrates ion behavior in beam modulation by moving colored dots, representing individual ions, across the screen of an IBM AT-compatible computer. (This program was used to ascertain the pattern of ion motion shown in Figure 4.) Then a simulation program was written to generate peak shapes for beam modulation TOFMS. The method used for this simulation will be explained with the aid of Figure 5. The program models the motion of various ions, chosen systematically from the region in-

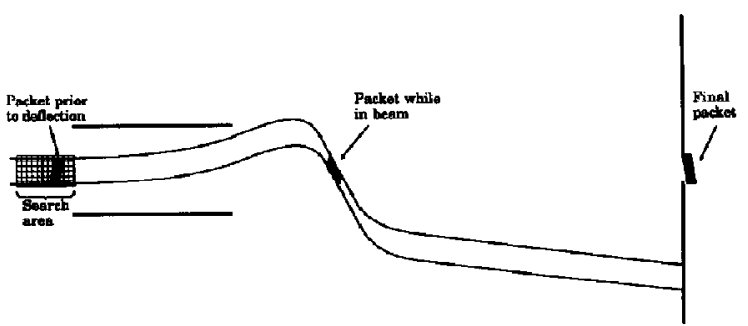

Figure 5. Illustration of ion behavior modeled by the simulation program (see text).

dicated, by means of Runge-Kutta integration through the deflection plate region and onward to the aperture. Flight times for those ions found to pass through the aperture are tabulated in the form of a histogram to generate the peak shape. The three solid regions in Figure 5 are meant to indicate the locus of points occupied by these ions at three instants in time: prior to entering the deflection region, part-way along the flight path, and just after passage through the aperture. (See ref 2 for a complete description of the shapes of these regions.)

An example of the output from this program is given in Figure 6 for the parameters shown in Table 1. The peak widths obtained by this simulation method may be approximated by means of eqs 1-3 by using calculated values of $w_{l}, w_{U}$, and $w_{\theta}$ to obtain estimates of peak variances. The variance of the overall peak is then given by the sum of the contributing variances. Examples of this process and comparisons with the simulated peaks are summarized in Table 2. Values for variances shown in parentheses were calculated as the difference between the other simulated values under the additivity-of-variances assumption. The peak

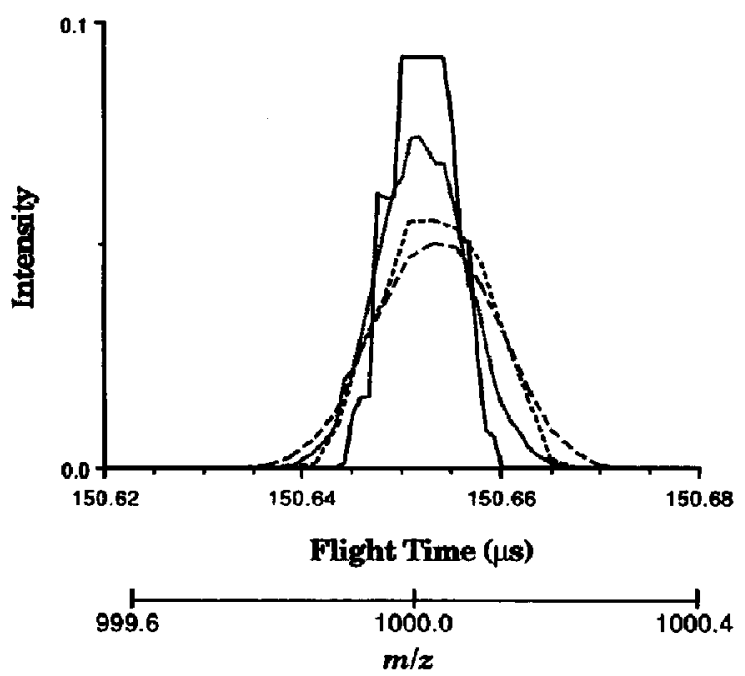

Figure 6. Simulated peak shape for $m / z$ 1000. $-=$ Bakker effect only;...$+=$ Bakker effect with energy dispersion; -...-. $=$ Bakker effect with angular dispersion; --- = all effects combined. 
Table 1. Parameters used for simulated beam deflection peak shape

\begin{tabular}{lc}
\hline$m$ & $1000 \mathrm{u}$ \\
$V_{\theta}$ & $500 . \mathrm{V}$ \\
$V_{d}$ & $100 . \mathrm{V}$ \\
$L$ & $1.500 \mathrm{~m}$ \\
$D$ & $0.005 \mathrm{~m}$ \\
$S$ & $0.005 \mathrm{~m}$ \\
$B$ & $0.003 \mathrm{~m}$ \\
$\theta$ & $0.1^{\circ}$ \\
$\beta$ & $0.0215 \mathrm{eV}$ \\
\hline
\end{tabular}

shape obtained by the Bakker effect alone may be approximated as a triangle. (This was found to yield more accurate results than a rectangular peak shape.) The variance of an isosceles triangle having a base width $w_{i}$ is given by $w_{1}^{2} / 24$. Similarly, the variance contributed by the angular effect may be approximated by $w_{\theta}^{2} / 12$; a rectangular peak shape is assumed here to model cases in which all values of $\theta$ within the limits given are equally likely. If the peak shape corresponding to the energy effect is assumed to be normal with a standard deviation of $w_{u} / 2.35$, the variance contributed by the energy effect is then $\left(w_{U} / 2.35\right)^{2}$. The accuracy of the simulated peak variances cannot be well characterized without, for example, detailed Monte Carlo analysis, but these widths are believed to fall within a few percent of the true values. Note that the prediction of peak widths from variance calculations is unreliable, in particular for the angular divergence effect. This is due to the variety of peak shapes (nearly rectangular to nearly Gaussian) that can occur as the various parameters are altered. For cases in which the angular divergence is very small (e.g., $\left.\theta<0.01^{\circ}\right)$, the predictions can be quite accurate; this was the case in the experimental study described below.

\section{Experimental Results}

A spectrum of perfluorotributylamine (PFTBA), obtained by direct inlet introduction with an ion source temperature of $150{ }^{\circ} \mathrm{C}$, is shown in Figure 7. These data result from the integration of 5000 transients col- lected in $1 \mathrm{~s}$ and summed by the integrating transient recorder (TTR) [14]. A comparison of peak basewidths for major ions in this spectrum to those produced by digital simulation is shown in Table 3 . For the simulated data, parameters matching the physical dimensions and voltages of the instrument were used, namely $L=2.1 \mathrm{~m}, S=6.35 \mathrm{~mm}, B=0.13$ $\mathrm{mm}, D=13 \mathrm{~mm}, V_{a}=3000 \mathrm{~V}$, and $V_{d}=63 \mathrm{~V}$. The standard deviation of the kinetic energy distribution, $\sigma_{U}$, was approximated as $0.5 \mathrm{eV}$ based on the potential distribution within the source; the angular divergence of the beam was neglected. The slope of base-width vs. $\sqrt{m / z}$ for the experimental values is calculated to be $3.7 \perp 0.2 \mathrm{~ns}$, and that for the simulated data is $4.0 \pm 0.1 \mathrm{~ns}$, demonstrating that the instrument performance follows the theory described above. An "expected" slope can be obtained by summing eqs 1 and 2 , then factoring out $m$. This procedure yields a value of $3.9 \mathrm{~ns}$ if $\beta$ is assigned a value of $6 \sigma_{U} / U_{0}$ to represent base-widths. This simple method is not appropriate when the angular divergence is large, but it is quite adequate for this case.

\section{Practical Considerations}

\section{Mass Resolving Power}

The PFTBA spectrum shown above was obtained with unit or better mass resolution up to at least $m / z 502$. An estimate of the upper mass limit for unit mass resolving power with this instrument can be found as follows. The flight time separation between ions of two adjacent $m / z$ values is given by

$$
\Delta t=\frac{L \mathbf{u}}{\sqrt{2 U}}(\sqrt{m / z+1}-\sqrt{m / z})
$$

where $u$ is the atomic mass unit. Equation 4 may be approximated by the first term of the Taylor series as

$$
\Delta t \approx \frac{L \sqrt{\mathbf{u}}}{2 \sqrt{2 U m / z}}
$$

In the previous section, the peak base width was found to be given approximately by $3.9 \sqrt{m / z}$ ns. (Either the experimental slope or the value found via simulation

Table 2. Comparison of peak variances obtained from calculation using eqs 1-3 and from digital peak shape simulation

\begin{tabular}{lccc}
\hline $\begin{array}{l}\text { Source of } \\
\text { peak broadening }\end{array}$ & $\begin{array}{c}\text { Calculated } \\
\text { base width } \\
\times 10^{-9} \mathbf{s}^{2}\end{array}$ & $\begin{array}{c}\text { Caiculated } \\
\text { variance } \\
\times 10\end{array}$ & $\begin{array}{c}\text { Simulation } \\
\text { variance } \\
\times 10^{-18} \mathbf{s}^{2}\end{array}$ \\
\hline Bakker effect & 13.6 & $\mathbf{8}$ & 9 \\
Energy & 1.7 & 11 & $111)$ \\
Angle & 8.9 & 7 & $116)$ \\
Bakker + energy & - & 19 & 20 \\
Bakker + angle & - & 15 & 25 \\
Bakker + energy + angle & - & 26 & 35 \\
\hline
\end{tabular}




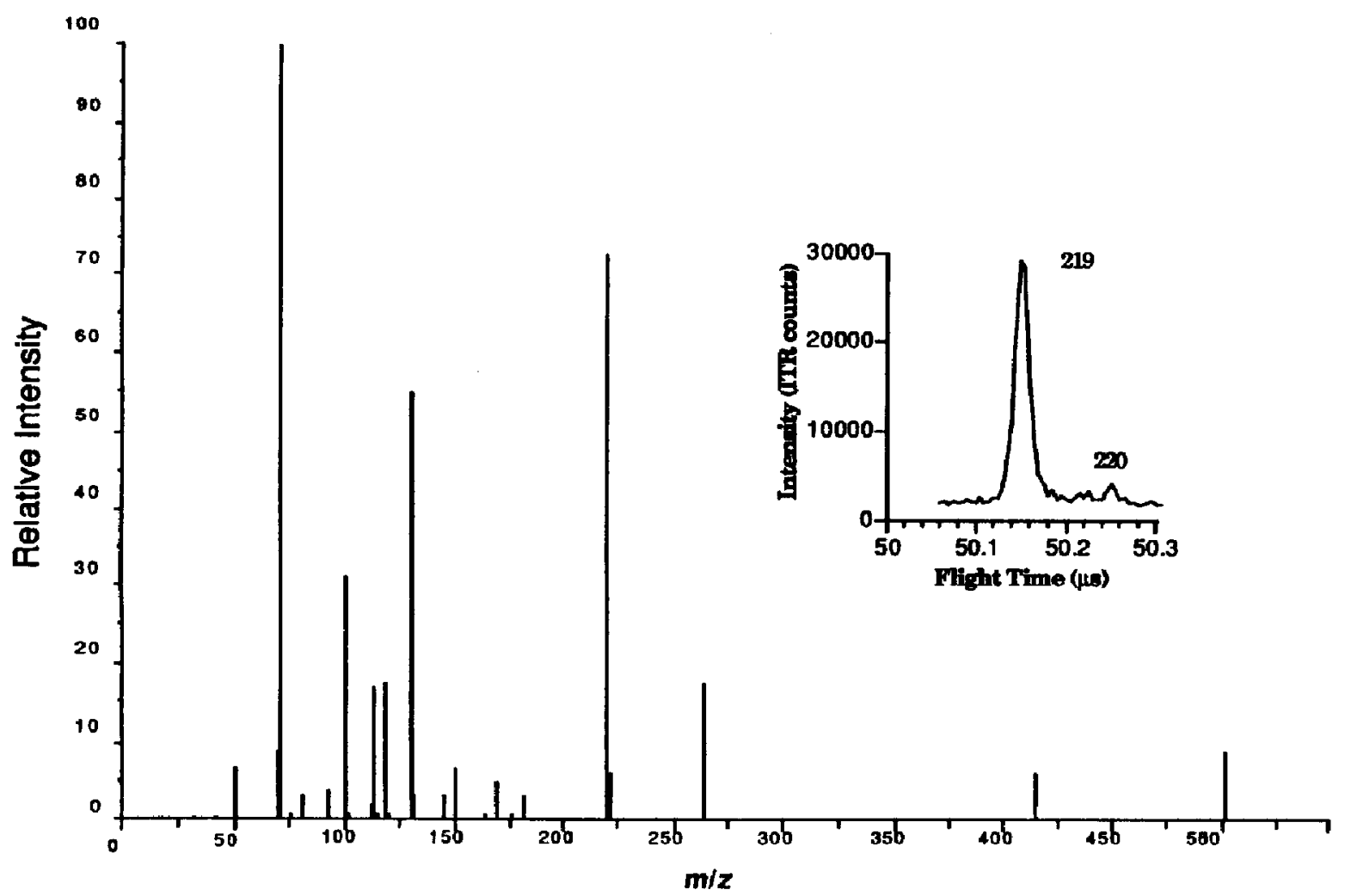

Figure 7. Mass spectrum of PFTBA collected by beam-deflection TOFMS. Typical peak shapes are shown in the inset for $m / z 219$ and $m / z 220$.

could also be used.) For simplicity, the width at halfheight (seconds) may be approximated as half of this value, thus

$$
w_{1 / 2} \approx 2.0 \times 10^{-9} \sqrt{m / z}
$$

Setting $\Delta t \approx w_{1 / 2}$ yields the $m / z$ value at which peak separation just equals peak width at half-height, and hence the approximate upper $m / z$ limit for unit mass resolving power. For the parameters in this example, this upper limit is an $\mathrm{m} / \mathrm{z}$ value of 710 .

An improvement could be made if an ion mirror were incorporated into the instrument. The ion mirror would remove the effect of the energy dispersion and, in addition, increase the effective flight path length

Table 3. Comparison of measured and simulated peak base widths

\begin{tabular}{rcc}
\hline$m / 2$ & Measured $( \pm 5 \mathrm{~ns})$ & Simulated $( \pm \mathbf{3 n s})$ \\
\hline \hline 69 & 40. & 38. \\
100 & 36. & 42. \\
131 & 52. & 46. \\
219 & 64. & 61. \\
264 & 67. & 69. \\
502 & 88. & 92. \\
\hline
\end{tabular}

by a factor of four. (The decreased flight time in the mirror contributes a factor of two, while the travel in the forward and reverse directions through the flight path contributes another factor of two.) In this case, the expected slope is obtained from eq 1 alone and has the value $2.5 \sqrt{m / z}$ ns. Using this value, and replacing $L$ by $4 L$ in eq 5 , the upper $m / z$ limit increases to 4400 . This is certainly adequate for most GC/MS applications.

\section{Signal-to-Noise Considerations}

It would appear that beam modulation in TOFMS would experience greatly reduced detectability and sensitivity due to the very small portion of the ion beam that is selected for detection. In addition, it would appear that the beam dimensions required for beam deflection, which are much smaller than those in conventional TOFMS, would further contribute to poor ion statistics. It is important to realize, however, that during the time an ion packet is striking the detector, the measured ion flux is the same as that seen from a continuous beam. The advantage of the continuous beam lies in the possibility of averaging the ion intensity measurements over a longer period of time. This advantage results in an improvement in signal-to-noise ratio $(S / N)$ equal to the square-root of the ratio of the measurement times. A TOF instrument with a packet 
width of $10 \mathrm{~ns}$ and an extraction repetition rate of 10 $\mathbf{k H z}$ would have an $\mathrm{S} / \mathrm{N} 100$ times worse than a continuous beam scanning instrument when used to monitor a single $m / z$ value. In practice, scanning sector mass spectrometers generally have much lower ion transmission efficiencies than TOF instruments due to the smaller apertures required throughout the flight path. In quadrupole mass spectrometers ion transmission is also limited by the acceptance angle and apertures required. Because scanning mass spectrometers act as mass-selective filters, the ion transmission generally decreases as the mass resolving power is increased. On the other hand, a TOFMS system that focuses (rather than filters) the ions can intensify the peak current rather than just reducing the peak width, as resolving power is increased. Because the ion mirror is a focusing device and, without grids, can be made with excellent (ca. 90\%) transmittance [15], the peak current in a mirror TOF instrument can be higher than the average instantaneous beam current for the same mass. These factors tend to counteract, to some degree, the decrease in S/N predicted from the duty cycle consideration alone.

In the previous paragraph, the ion intensity at the peak arrival time for an ion of a particular $\mathrm{m} / \mathrm{z}$ in a TOF instrument is compared with the peak ion intensity for that same $m / z$ in a mass scanning instrument that is fixed at the optimum setting for the $m / z$ being monitored (selected-ion-monitoring, or SIM, mode). To compare TOF and scanning instruments for modes in which full spectra are generated, other factors need to be considered. In scanning mode, the scanning instrument monitors a single $m / z$ window at a time. The average time spent at each window is equal to the scan time divided by the number of mass values sampled, assuming no dead time between values. With TOFMS using boxcar integrator detection (sometimes called time-slice detection), the output signal also represents the intensity of only one arrival time window at a time. The delay time for the sample window of the boxcar integrator is stepped or scanned to obtain a mass spectrum just as in the scanning filter mass spectrometers. For this type of detection, the relative $\mathrm{S} / \mathrm{N}$ for both scanning and TOF mass spectrometers would be equal to that discussed above for the SIM mode. The duty cycle disadvantages of TOF are offset, at least partially, however, by its transmittance and focusing advantages.

A very significant advantage can be seen for TOF, however, when time-array detection is performed using an ITR. The ITR acquires, for all time slices within the collection period, the same information the boxcar integrator does for only one. For an ITR that stores 8192 samples per transient, the effect is an $S / \mathrm{N}$ improvement of $\sqrt{\mathbf{8 1 9 2}} \approx 91$ times that of the scanning boxcar data collection system. Because the scanning boxcar data system has, at worst, a 100-times lower $\mathrm{S} / \mathrm{N}$ than the scanning filter instrument, the TOF spectrometer with an ITR and mirror should have an $\mathrm{S} / \mathrm{N}$ at least equal to and potentially much better than a scanning filter instrument in scanning mode. The increased data rate available with TOFMS with ITR detection can be used to great advantage when spectra are to be collected under conditions of rapidly changing sample pressure in the source such as encountered with high-performance chromatography.

\section{Conclusions}

Beam deflection is becoming a common technique in TOFMS, yet beam deflection systems are often designed with the assumption that the ion behavior so produced will follow the Gate model. For optimum time-resolution, however, the more complex Differential Impulse Sweep mechanism is required. This study showed that accurate predictions of ion behavior under this model can be obtained either by simple inspection of the equations given or by digital simulation of ion motion. Such predictions can be of assistance in the design of beam deflection TOFMS instruments. The study also demonstrated that a beam deflection TOFMS instrument incorporating an ion mirror and an ITR would have mass resolution and sensitivity suitable for modern GC/MS applications.

\section{Acknowledgments}

This work was supported by a grant from the Biotechnology Research Program of the National Institutes of Health Division of Research Kesources (DRR-00480-20). The authors would like to thank M. Rabb and R. Tecklenburg for their assistance.

\section{References}

1. Fowler, T. K.; Good, W. M. Nucl. Instrum. Meth. 1960, 7, 245.

2. Bakker, J. M. B. J. Phys. E: Sci. Instrum. 1973, 6, 785.

3. Bakker, J. M. B. J. Phys. E: Sci. Instrum. 1974, 7, 364.

4. Pinkston, J. D.; Rabb, M.; Watson, J. T,; Allison, J. Rev. Sci. Instrum. 1986, 57, 583.

5. Glish, G. L.; Goeringer, D. E. Anal. Chem. 1984, 56, 2291.

6. Eckenrode, B. A.; Watson, J. T.; Enke, C. G.; Holland, J. F. Int. J. Mass Spectrom. Ion Proc. 1988, 83, 177.

7. Futrell, J. H.; Tiernan, T. D.; Abramson, F. P.; Miller, C. D. Rev. Sai. Instrum. 1968, 39, 340.

8. Miller, C. D.; Tiernan, T. D.; Futrell, J. H. Rev. Sci. Instrum. 1969, 40, 503.

9. Schey, K.; Cooks, R. G.; Grix, R.; Wollnik, H. Int. J. Mass Spectrom. Ion Proc. 1987, 77, 49.

10. Dawson, I. H. J.; Guilhaus, M. Rapid Commun. Mass Spectrom. 1989. 3, 155.

11. Yefchak, G. E.; Enke, C. G.; Holland, J. F. Int. J. Mass Spectrom. Ion Proc. 1989, 87, 313.

12. Wiley, W. C.; McLaren, I. H. Rev. Sci. Instrum. 1955, 26, 1150.

13. Stein, R. Int. J. Mass Spectrom. Ion Phys. 1974, 14, 205.

14. Holland, J. F.; Newcome, B.; Tecklenburg, R. E. Jr.; Davenport, M.; Altison, J.; Watson, J. T.; Enke, C. G. in preparation.

15. Hermann Wollnik, personal communication, 1988. 